\title{
Remission of renal disease: recounting the challenge, acquiring the goal
}

\author{
Barry M. Brenner \\ Brigham and Women's Hospital, Harvard Medical School, Boston, Massachusetts, USA \\ J. Clin. Invest. 110:1753-1758 (2002). doi:10.1172/JCI200217351.
}

Renal excretory function begins at the glomerular capillaries with the formation of a nearly ideal ultrafiltrate of plasma. The volume and composition of the ultrafiltrate undergo sequential change along the nephron, ultimately regulated to preserve near-constancy of mineral, electrolyte, and fluid balance. Modest derangement of these processes often escapes clinical or laboratory detection, because the myriad of unimpaired nephrons compensate more or less completely. Beyond a certain level of injury, however, compensatory adaptations no longer keep pace with nephron loss. As a result, glomerular filtration rate declines and organic nitrogenous wastes (e.g., creatinine, urea) accumulate in plasma and other body fluids. In acute renal failure, injury is usually transient, so that deterioration of function is short-lived and reversible. Not so, however, with the many forms of irreversible nephron damage, which typically progress over time, often eventuating in complete loss of renal function, systemic toxicity (uremia), and death unless the lost function is replaced by chronic dialysis and/or successful renal transplantation. Much of the effort in nephrology since the 1970s has been concerned with improving the effectiveness of these renal-replacement therapies. In the late 1960s, we fortuitously gained access to a unique strain of rats with many glomeruli that are situated on the renal cortical surface. For the first time, mammalian glomeruli were therefore accessible to direct study by new microtechniques developed in our laboratory. We therefore addressed the following questions: (a) What are the precise hemodynamic forces and biophysical properties that govern glomerular capillary function in health?

\footnotetext{
Address correspondence to: Barry M. Brenner, Renal Division, Brigham and Women's Hospital, 75 Francis Street, Boston, Massachusetts 02115-6195, USA. Phone: (617) 732-5850; Fax: (617) 732-6392; E-mail: bbrenner@partners.org.

Conflict of interest: The author has declared that no conflict of interest exists.

Nonstandard abbreviations used: end-stage renal disease (ESRD); angiotensin-converting enzyme inhibitor (ACEI); angiotensin receptor blocker (ARB); renin-angiotensin system (RAS); Irbesartan Type 2 Diabetic Nephropathy Trial (IDNT); Reduction of Endpoints in NIDDM [non-insulin-dependent diabetes mellitus] with the Angiotensin II Antagonist Losartan (RENAAL); Ramipril Efficacy in Nephropathy (REIN); Heart Outcomes Prevention Evaluation (HOPE).
}

(b) How are these elements modified during renal injury? (c) Do these modifications contribute to the relentless progression of renal disease? (d) If so, can they be reversed, so as to retard further deterioration of renal function and prevent end-stage renal disease (ESRD)?

\section{Mechanisms of normal glomerular function}

In 1843, Ludwig proposed that the initial event in the process of urine formation is the production of an ultrafiltrate of plasma across the glomerular capillary wall (1). Starling (2) further suggested that the mechanisms responsible for glomerular ultrafiltration are the same as those governing the movement of fluid across all other capillaries - namely, the magnitude and direction of the imbalance of transcapillary hydraulic and colloid osmotic pressures. These concepts initially received direct experimental confirmation in nonmammalian species, because in mammals glomeruli are rarely present as surface structures on the kidney and are therefore inaccessible to direct study. In the late 1960s, however, a strain of Wistar rats with some glomeruli situated on the renal surface was identified in the laboratory of Klaus Thurau of the University of Munich, who kindly shipped a dozen of these rats to my laboratory in San Francisco aboard a Lufthansa 747 jumbo jet. (That only eleven were in the cage upon arrival led me to avoid flying on this airline for fear that essential cables might have been gnawed by the escapee.)

The second key development that enabled us to make direct in vivo measurements of mammalian glomerular capillary hydraulic pressure occurred in the mid-1960s when Wiederhielm and colleagues designed and built a sensitive, servo-null microtransductor system suitable for continuous measurement of microcirculatory pressures (3). Cleaves Bennett, then the senior fellow in the late Robert W. Berliner's laboratory at the NIH, recognized the potential value of this instrument for the online study of the renal microcirculation and commissioned the NIH technical staff in 1968 to construct a similar device from Wiederhielm's kindly supplied blueprints. Fortunately for me, a more junior fellow in Berliner's lab, Bennett took a position at Duke University before the NIH device was completed, leaving the new instrument for Julia Troy, my technical assistant, and me to deploy, initially in studies of peritubular capillary control of proximal tubule fluid reabsorption. 
Soon thereafter, Troy and I moved to San Francisco and transferred the instrument to another government facility, our new lab at the Fort Miley Veterans Administration Hospital.

Within a very few days of obtaining these unique rats (which we dubbed Munich-Wistar rats), some were set aside to breed and others quickly yielded the first highly reproducible values for glomerular pressure approximately $45 \mathrm{mmHg}$, or only about one-half of mean arterial pressure, rather than the 90- to 100 $\mathrm{mmHg}$ value postulated by others previously. Together with simultaneous measurements of single-nephron GFR and pre- and postglomerular capillary protein concentrations (which enabled calculation of colloid osmotic pressures), we were able to describe the entire net force profile for filtration along the capillary network of a single glomerulus. We concluded that net filtration pressure is at maximum at the afferent arteriolar end of the glomerulus $(\sim 15 \mathrm{mmHg})$ and dissipates to near 0 by the efferent end. In consequence, GFR is highly plasma flow-dependent, and the ultrafiltration coefficient is far above values estimated for other filtering capillary beds.

I wrote a manuscript summarizing these initial findings in 2 days, had it reviewed by my coworkers on the third day, and mailed it to the JCI on the fourth day. The article was accepted without revision three weeks later (4). Three decades and several hundred articles later, this good fortune has not recurred!

The ability to make measurements of the critical determinants of glomerular filtration created an enormous opportunity for extending our understanding of glomerular function in health and disease. A major step forward came from an alliance with Channing Robertson, a young faculty member in the Department of Chemical Engineering at nearby Stanford University, and his eager graduate students, William Deen, Ramsay Chang, and Michael Bohrer. We developed a mathematical model of glomerular ultrafiltration (5) and confirmed a series of functional predictions in followup studies (the so-called Glomerular Dynamics series) of autoregulatory mechanisms, quantification of the ultrafiltration coefficient, responses to ischemic and ablative renal injury, and extension of servo-null methodology to the kidney of the primate $(6-13)$.

From these early studies came evidence that the glomerular capillary ultrafiltration coefficient $\left(K_{\mathrm{f}}\right)$ could be modified by vasoactive substances such as angiotensin II, giving rise to the hypothesis that contractile cells in the glomerulus must govern $K_{\mathrm{f}}$ by altering net filtration surface area $(14,15)$. Mesangial cells seemed ideally suited to this task, an impression soon confirmed in in vitro studies of mesangial cell contractility and planar surface area under conditions of exposure to a variety of vasoactive substances. In large measure, the subsequent intense interest in the biology of various glomerular cells in vitro derived from this hypothesis.

The ability to quantify filtration kinetics also laid the groundwork for a series of studies of the permselectivity properties of the glomerular capillary wall, i.e., the discriminatory function of the wall with respect to transmural passage of molecules of varying size, charge, and shape (16-22). Discovery of charge-selectivity and shape-selectivity greatly enhanced our understanding of glomerular sieving phenomena and added powerful explanatory capability to the interpretation of clinical proteinuria (23).

Yet another major insight from these early in vivo studies of glomerular function came from the finding that partial renal ablation leads to adaptive increases in hydraulic pressure in surviving glomeruli $(24,25)$. It soon became evident that glomerular pressure elevations also occur when nephron number is reduced by immune, ischemic, or chemical injury, suggesting a reciprocal relationship between nephron number and glomerular blood pressure (26). Insofar as raised glomerular capillary pressure proved to be a powerful predictor of subsequent glomerular fibrotic injury, the field was ripe for a test of this prediction - specifically, does prevention of the adaptive rise in glomerular pressure abrogate subsequent progressive renal injury? A series of studies was performed in Munich-Wistar rats in which dietary protein restriction and the pharmacologic amelioration of glomerular hypertension indeed arrested or dramatically slowed disease progression; drugs that preferentially prevented angiotensin IIinduced glomerular and postglomerular vasoconstriction were especially effective.

\section{Mechanisms of progression of chronic renal disease: identifying the final common pathway}

When GFR in humans falls below about half of normal, further loss of function commonly ensues, even when the original disease becomes inactive. In response to reduced renal mass, surviving nephrons undergo adaptations in structure and function that raise singlenephron GFR to meet excretory demands. To our surprise, these glomerular hemodynamic adaptations responsible for increased single-nephron GFR actually proved to initiate and perpetuate glomerular injury following experimental renal mass ablation, suggesting that similar events may occur when nephrons are lost through disease (24-26). In addition to the detrimental effects of acquired nephron loss, inborn deficits in total nephron supply were also considered a likely cause of progressive glomerular injury and hypertension in later life (27).

The most unfavorable glomerular hemodynamic adaptation to congenital deficits or focal nephron obliteration by disease is elevated glomerular capillary pressure, which ultimately leads to glomerular scarring and nephron dropout. Among a variety of measures that slow progression of experimental renal disease, alleviation of glomerular capillary hypertension was found to be the common denominator. Glomerular capillary hypertension is often maintained by angiotensin-dependent mechanisms, via increased systemic blood pressure and efferent arteriolar vasoconstriction. In addition to their potent systemic antihypertensive actions, angiotensinconverting enzyme inhibitors (ACEIs) and angiotensin receptor blockers (ARBs) are highly effective in 
controlling glomerular capillary hypertension and thereby in retarding disease progression (28). In rats with experimental renal disease, we observed that, for an equivalent systemic blood pressure-lowering effect, ACEIs reduced glomerular capillary pressure and slowed the rate of renal disease progression, whereas, with a combination of hydralazine, reserpine, and a diuretic, despite equivalent systemic blood-pressure lowering, glomerular hypertension persisted and disease progression continued unabated (29). Subsequent studies consistently demonstrated renal protection with inhibitors of the reninangiotensin system (RAS) in a variety of experimental models of renal injury (28).

Although angiotensin II has emerged as a central mediator of the glomerular hemodynamic changes associated with progressive renal injury, several nonhemodynamic effects of angiotensin II may also be important in renal disease progression (Figure 1). These include production of reactive oxygen species; coordinated upregulation of cytokines, cell adhesion molecules and profibrotic growth factors, which in turn give rise to mesangial cell proliferation; induction of TGF- $\beta$ expression; increased synthesis of ECM proteins; stimulation of plasminogen activator inhibitor-1 production by endothelial and vascular smooth muscle cells; and macrophage activation and infiltration (30). Angiotensin II also augments the adrenal production of aldosterone, a recently recognized contributor to renal injury (31), and augments glomerular transcapillary passage of plasma proteins, the principal cause of proteinuria.

Proteinuria has traditionally been regarded as a marker of glomerular filtration barrier integrity, and the extent of proteinuria has therefore been used as an indicator of glomerular disease severity. Recent evidence suggests that proteinuria also contributes to progressive renal injury. Experimental observations suggest mechanisms whereby an excess of filtered proteins may promote renal damage. Growing tubule epithelial cells in the presence of a variety of plasma proteins in vitro induces the production of proinflammatory cytokines and ECM proteins, responses that ultimately contribute to glomerular scarring and tubulointerstitial fibrosis (Figure 1) (32). In vivo, proteinuria is associated with the renal expression of cell adhesion molecules and chemoattractants, forerunners of tubulointerstitial inflammation and fibrosis $(32,33)$. Together, these data support the hypothesis that excessive filtration of serum proteins by injured glomeruli contributes directly to progressive renal damage.

Figure 1

\section{Clinical trials of renoprotection:} translation from bench to clinic

Dietary approaches. More than 50 years ago, Addis (34) speculated that the severity of renal disease could be ameliorated by reducing the excretory burden for nitrogen through dietary protein restriction. Our early studies in the partial nephrectomy model revealed that dietary protein restriction abrogates the adaptive rise in glomerular pressure and thereby slows the tendency to renal disease progression (24). Despite the longevity of this hypothesis and unambiguous support from experimental studies, confirmation of a beneficial effect of protein restriction in clinical trials has proved elusive. However, a recent meta-analysis of ten randomized, controlled studies of the effects of protein restriction on the progression of diabetic and nondiabetic renal disease determined that the overall relative risk of renal failure or death was indeed reduced with protein restriction, as compared with nonrestricted protein intake (35). Additional support for this conclusion has recently been reported $(36,37)$.

Pharmacologic approaches: diabetic nephropathy. Our studies, in rodents, showing that control of glomerular capillary hypertension with ACEIs retards the development of the glomerular lesions of experimental diabetic nephropathy (38) soon motivated a number of clinical trials. Results of several small clinical studies performed to assess the effects of antihypertensive treatment in general, and ACEIs in particular, on the rate of progression of diabetic nephropathy were reviewed by Mogensen (39). Whereas many of these studies appeared to show a favorable response to therapy, none

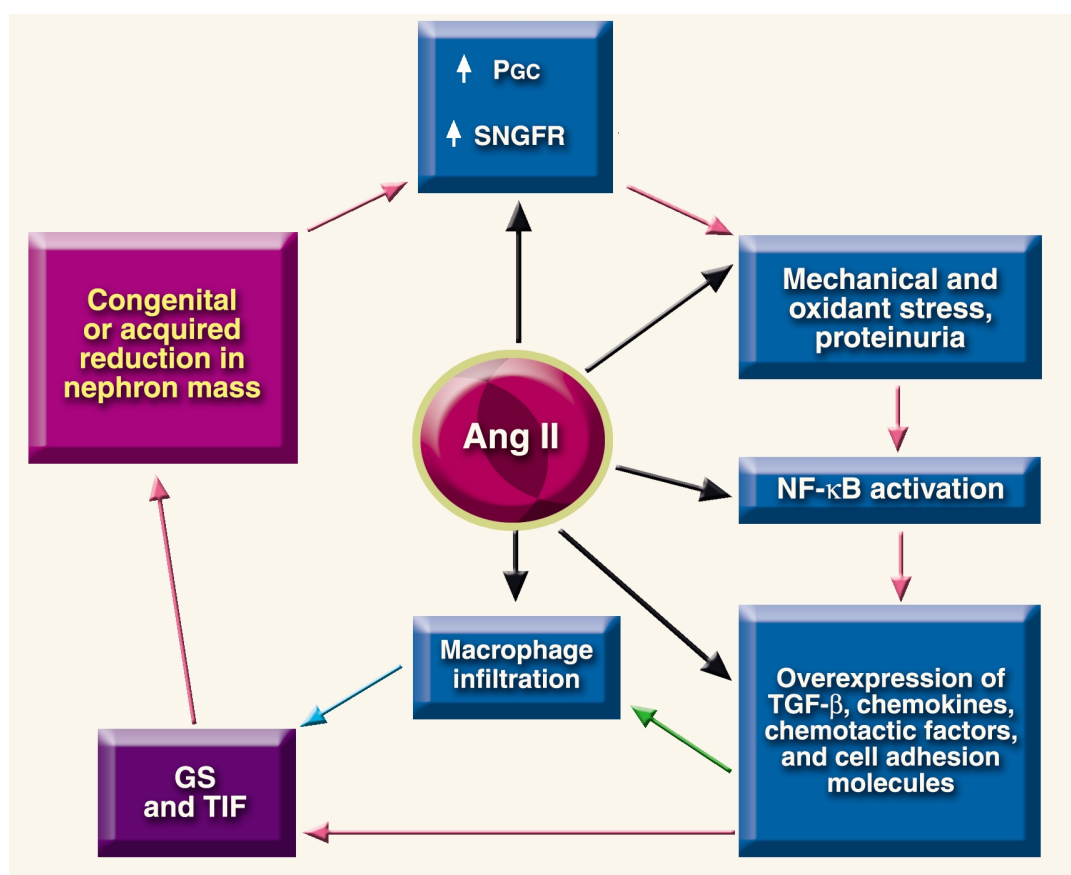

Final common pathway for progression of chronic renal disease. Angiotensin II (ang II) promotes injury in at least five separate steps in the cycle. $\mathrm{P}_{\mathrm{GC}}$, Glomerular capillary pressure; SNGFR, single-nephron GFR; GS, glomerulosclerosis; TIF, tubulo-interstitial fibrosis. 
was sufficiently robust statistically to conclusively establish the value of antihypertensive treatment or ACEIs in diabetic nephropathy. These shortcomings were resolved by the clinical trial entitled "The effect of angiotensin-converting-enzyme inhibition on diabetic nephropathy" (40). Four hundred seven patients with type 1 diabetes and proteinuria (>500 $\mathrm{mg} / \mathrm{d}$ ) were randomized to receive either the ACEI captopril or placebo. Blood pressure was managed independently of the experimental treatment, using agents other than ACEIs or calcium channel blockers. Patients receiving captopril were, on average, only $48 \%$ as likely to double their serum creatinine as were those receiving placebo. Captopril treatment was also associated with a $50 \%$ reduction in the combined risk of death, dialysis, or transplantation. These striking results provided solid clinical evidence for effective retardation of nephropathy, in this case due to type 1 diabetes, and led to the first federally approved treatment in the US for slowing the progression of renal disease.

It should be noted, however, that most diabetic patients who develop ESRD suffer from type 2 diabetes, reflecting its approximately 20 -fold greater prevalence over type 1 . Type 2 diabetic patients develop glomerular hyperfiltration, proteinuria, and progressive declines in GFR, much as in type 1 diabetes and with essentially the same time course. Renal protection with ACEIs was observed by Ravid et al. in a small multicenter, doubleblind, randomized controlled trial that compared the effects of enalapril with those of placebo over 7 years in 94 normotensive type 2 diabetics with microalbuminuria and normal renal function (41). Enalapril treatment was associated with stable microalbuminuria over the 7-year follow-up, whereas microalbuminuria increased roughly twofold in the placebo group. GFR was estimated to decline progressively in the placebo group, reflecting a $16 \%$ loss at 7 years, but remained stable at base-line levels in those receiving enalapril. Subsequently, Kasiske et al. performed a meta-analysis of studies involving 2,494 patients and also concluded that ACEIs were uniquely renoprotective (42).

ARBs inhibit the RAS by blocking angiotensin II subtype $1\left(\mathrm{AT}_{1}\right)$ receptors. Thus, whereas ACEIs inhibit angiotensin-converting enzyme-dependent angiotensin II production, ARBs block the effects of angiotensin II from any source at the receptor level. Despite these differences in mechanisms of action, experimental studies reveal that ACEIs and ARBs produce similar improvements in glomerular hemodynamics and afford equivalent renoprotection in a variety of experimental models of renal disease (28).

Two large, recently completed, prospective, multicenter, randomized trials showed that interruption of the RAS with ARBs in type 2 diabetic subjects with overt nephropathy delays the progression of renal disease $(43,44)$. The Irbesartan Type 2 Diabetic Nephropathy Trial (IDNT) evaluated the effects of the ARB irbesartan on renal and cardiovascular morbidity and mortality versus the effects of conventional therapy (placebo group) or the calcium channel blocker amlodipine in 1,715 subjects (43). The primary composite endpoint of the study was the time to a first event - doubling of base-line serum creatinine, ESRD (renal transplantation, need for dialysis, or serum creatinine $\geq 530 \mu \mathrm{mol} / 1[6.0 \mathrm{mg} / \mathrm{dl}]$ ), or death (all-cause mortality). The secondary composite endpoint was again the time to a first event - cardiac fatality, nonfatal myocardial infarction, or hospitalization for heart failure, stroke, above-the-ankle amputation, or revascularization (cardiac, carotid, or peripheral vascular). For subjects receiving irbesartan, the adjusted relative risk of reaching the primary composite endpoint was $19 \%$ lower than for those receiving placebo $(P=0.02)$ and $23 \%$ lower than for those receiving amlodipine $(P=0.006)$. The relative risk of doubling of serum creatinine in the irbesartan group was $29 \%$ lower than that in the placebo group $(P=0.009)$ and $39 \%$ lower than that in the amlodipine group $(P<0.001)$. There was no significant difference between placebo and amlodipine for the primary composite endpoint. The relative risk of ESRD in the irbesartan group was $17 \%$ lower than that in the placebo group and $24 \%$ lower than that in the amlodipine group, but these differences did not achieve statistical significance. Secondary cardiovascular outcomes also failed to show significant differences among the various arms of the IDNT study. Proteinuria was reduced an average of $33 \%$ in the irbesartan arm, compared with $6 \%$ and $10 \%$ in the amlodipine and placebo arms, respectively. The more favorable renal outcomes in the irbesartan group were in excess of effects directly attributable to blood pressure control.

The Reduction of Endpoints in NIDDM [non-insulin-dependent diabetes mellitus] with the Angiotensin II Antagonist Losartan (RENAAL) study was undertaken to determine whether losartan reduces the number of patients with type 2 diabetes that experience a doubling of serum creatinine, ESRD, or death, as compared with placebo-treated subjects (44). The primary and secondary endpoints of the study were similar to those in the IDNT study, but treatment was of longer average duration in the RENAAL study (3.4 vs. 2.6 years). In RENAAL, 1,513 subjects were randomized to receive either losartan or placebo once daily on a background of conventional antihypertensive therapy, excluding ACEIs and ARBs. Losartan treatment reduced the relative risk of the primary composite endpoint by $16 \%(P=0.024)$. The risk of doubling of serum creatinine, the risk of ESRD, and the combined risk of ESRD or death were decreased by $25 \%(P=0.002), 28 \%(P=0.006)$, and $20 \%(P=0.010)$, respectively. RENAAL is thus the only study to date to specifically reduce the risk of ESRD in diabetes, in this case with losartan. Proteinuria declined by $34 \%$ in the losartan arm and increased slightly in the placebo group $(P=0.001)$, and losartan slowed the estimated rate of loss of GFR by $18 \%$ relative to placebo. No significant difference was observed between the losartan and placebo arms for the secondary composite endpoint of cardiovascular morbidity and mortality, or for most of the cardiovascular components, although the losartan arm showed a significant reduction of $32 \%$ $(P=0.005)$ in the risk of a first hospitalization for 
heart failure. Once again, these consistent benefits of losartan in the RENAAL study were above and beyond effects that could be attributed to measured reductions in blood pressure.

Pharmacologic approaches: nondiabetic nephropathy. Several studies investigated the potential of ACEIs to afford renoprotection in nondiabetic forms of clinical renal disease. Maschio et al. randomly assigned 583 patients with renal disease of diverse etiologies to treatment with benazepril or placebo (45). After 3 years of follow-up, the study found a 53\% reduction, with ACEI treatment, in the combined risk of doubling of the base-line serum creatinine or need for dialysis. However, a significantly lower blood pressure among patients receiving ACEI versus placebo made it difficult to separate the beneficial effects of blood pressure reduction from any unique renoprotective effects of ACEIs.

In the more recent Ramipril Efficacy in Nephropathy (REIN) study, 352 patients with nondiabetic renal disease, randomly assigned to receive either ACEIs or placebo, achieved similar control of blood pressure (46). Among patients with proteinuria of at least $3 \mathrm{~g} / \mathrm{d}$ at base line, a significantly lower rate of decline in GFR was seen in patients receiving ACEIs ( 0.53 vs. $0.88 \mathrm{ml} / \mathrm{min} / \mathrm{mo})$. In a second phase of the study, patients who initially received placebo were switched to ACEIs, and those already on ACEIs continued this treatment $(47,48)$. Consistent with the findings in the first 2-year phase of the study, those switched from placebo to ACEIs enjoyed a significant reduction in the rate of decline in GFR, while patients continuing on ACEI treatment enjoyed a further reduction in the rate of GFR decline, to levels similar to those associated with normal aging. Indeed, from 36 to 54 months of follow-up, no patients in the latter group reached ESRD, and a small number actually experienced a rise in GFR (48). One hundred eighty-six other REIN-study patients with less than $3 \mathrm{~g} / \mathrm{d}$ of proteinuria at base line also benefitted from ACEIs with reduced incidence of ESRD, particularly those with a GFR of less than $45 \mathrm{ml} / \mathrm{min}$ at base line (49).

A recent patient-based meta-analysis of 1,860 nondiabetic subjects from 11 randomized ACEI-versusplacebo-treatment trials also concluded that ACEIs are more effective than other antihypertensive treatment regimens in slowing disease progression and reducing proteinuria (50). A similar conclusion emerged from the African-American Study of Kidney Disease (AASK) trial in hypertensive African-Americans, in which ramipril proved more renoprotective than the comparator drugs, amlodipine or metoprolol (51). In addition to the renoprotective effects of ACEI treatment, the recent Heart Outcomes Prevention Evaluation (HOPE) study reported a substantial reduction in allcause and cardiovascular mortality with ramipril versus placebo among 9,297 patients who were at increased risk of cardiovascular events (52). Because cardiovascular disease is the single largest cause of morbidity and mortality among patients with chronic renal disease, the HOPE study data provide a further rationale for the use of drugs that interrupt the RAS in patients with renal disease.
Large randomized clinical studies of the renoprotective effects of ARBs in nondiabetic renal disease are still awaited, but preliminary data suggest that ARBs are likely to be as effective as ACEIs. In small studies, ARBs and ACEIs produced similar antihypertensive and antiproteinuric effects in patients with essential hypertension or chronic renal disease. One important advantage of ARBs over ACEIs is their more favorable sideeffect profile, as ARBs are not associated with the cough that may occur in up to $40 \%$ of patients receiving ACEIs. Finally, the differing effects of ACEIs and ARBs on the RAS imply that in combination they may have additive or even synergistic effects, and early evidence appears to support this contention (53).

\section{Summary}

In less than two decades, the use of ACEIs and ARBs as therapeutic interventions for slowing renal disease progression has made the giant leap from laboratory to universal clinical practice. In all likelihood, other novel renoprotective agents will emerge from future laboratory and clinical studies, but it is already clear that currently available strategies not only delay the need for dialysis but may actually prevent many patients from ever progressing to ESRD. It may be worth emphasizing that while the original studies from my laboratory centered upon basic issues in glomerular capillary physiology, it soon became evident that our findings might shed light on mechanisms of renal disease progression and on rational approaches to their interruption. That this has come to pass serves to reinforce the important and unique role played by physician-scientists in the pursuit of fundamental and initially untargeted biomedical research.

1. Ludwig, C. 1843. Beitrage zur lehre com mechanismus der harnsekretion. Elwert'sche universitats: und verlagsbuchhandlun. Marburg, Germany. $42 \mathrm{pp}$.

2. Starling, E.H. 1899. The glomerular functions of the kidney. J. Physiol. 24:317-330.

3. Wiederhielm, C.A., Woodbury, J.W., Kirk, S., and Rushmer, R.F. 1964. Pulsatile pressures in the microcirculation of frog's mesentery. Am. J. Physiol. 207:173-176.

4. Brenner, B.M., Troy, J.L., and Daugharty, T.M. 1971. The dynamics of glomerular ultrafiltration in the rat. J. Clin. Invest. 50:1776-1780.

5. Deen, W.M., Robertson, C.R., and Brenner, B.M. 1972. A model of glomerular ultrafiltration in the rat. Am. J. Physiol. 223:1178-1183.

6. Brenner, B.M., Troy, J.L., Daugharty, T.M., Deen, W.M., and Robertson, C.R. 1972. Dynamics of glomerular ultrafiltration in the rat. II. Plasmaflow dependence of GFR. Am. J. Physiol. 223:1184-1190.

7. Robertson, C.R., Deen, W.M., Troy, J.L., and Brenner, B.M. 1972. Dynamics of glomerular ultrafiltration in the rat. III. Hemodynamics and autoregulation. Am. J. Physiol. 223:1191-1200.

8. Deen, W.M., Troy, J.L., Robertson, C.R., and Brenner, B.M. 1973. Dynamics of glomerular ultrafiltration in the rat. IV. Determination of the ultrafiltration coefficient. J. Clin. Invest. 52:1500-1508.

9. Daugharty, T.M., Ueki, I.F., Mercer, P.F., and Brenner, B.M. 1974. Dynamics of glomerular ultrafiltration in the rat. V. Response to ischemic injury. J. Clin. Invest. 53:105-116.

10. Maddox, D.A., Deen, W.M., and Brenner, B.M. 1974. Dynamics of glomerular ultrafiltration. VI. Studies in the primate. Kidney Int. 5:271-278

11. Deen, W.M., Maddox, D.A., Robertson, C.R., and Brenner, B.M. 1974. Dynamics of glomerular ultrafiltration in the rat. VII. Response to reduced renal mass. Am. J. Physiol. 227:556-562.

12. Myers, B.D., Deen, W.M., Robertson, C.R., and Brenner, B.M. 1975. Dynamics of glomerular ultrafiltration in the rat. VIII. Effects of hematocrit. Circ. Res. 36:425-435.

13. Baylis, C., Ichikawa, I., Willis, W.T., Wilson, C.B., and Brenner, B.M. 1977. Dynamics of glomerular ultrafiltration. IX. Effects of plasma protein concentration. Am. J. Physiol. 232:F58-F71. 
14. Myers, B.D., Deen, W.M., and Brenner, B.M. 1975. Effects of norepinephrine and angiotensin II on the determinants of glomerular ultrafiltration and proximal tubule fluid reabsorption in the rat. Circ. Res. 37:101-110.

15. Baylis, C., Deen, W.M., Myers, B.D., and Brenner, B.M. 1976. Effects of some vasodilator drugs on transcapillary fluid exchange in renal cortex. Am. J. Physiol. 230:1148-1158.

16. Chang, R.L.S., Robertson, C.R., Deen, W.M., and Brenner, B.M. 1975. Permselectivity of the glomerular capillary wall to macromolecules. I. Theoretical considerations. Biophys. J. 15:861-886.

17. Chang, R.L.S., Ueki, I.F., Troy, J.L., Deen, W.M., and Brenner, B.M. 1975 Permselectivity of the glomerular capillary wall to macromolecules. II. Experimental studies in rats using neutral dextran. Biophys. J. 15:887-906.

18. Chang, R.L.S., Deen, W.M., Robertson, C.R., and Brenner, B.M. 1975. Permselectivity of the glomerular capillary wall. III. Restricted transport of polyanions. Kidney Int. 8:212-218.

19. Deen, W.M., Robertson, C.R., and Brenner, B.M. 1974. Concentration polarization in an ultrafiltering capillary. Biophys. J. 14:412-431.

20. Chang, R.L.S., Deen, W.M., Robertson, C.R., Bennett, C.M., and Brenner, B.M. 1976. Permselectivity of the glomerular capillary wall. Studies of experimental glomerulonephritis in the rat using neutral dextran. J. Clin. Invest. 57:1272-1286.

21. Bennett, C.M., et al. 1976. Permselectivity of the glomerular capillary wall. Studies of experimental glomerulonephritis in the rat using dextran sulfate. J. Clin. Invest. 57:1287-1294.

22. Brenner, B.M., Baylis, C., and Deen, W.M. 1976. Transport of molecules across renal glomerular capillaries. Physiol. Rev. 56:502-534

23. Deen, W.M., Bridges, C.R., Brenner, B.M., and Myers, B.D. 1985. Heteroporous model of glomerular size-selectivity: application to normal and nephrotic humans. Am. J. Physiol. 249:F374-F389.

24. Hostetter, T.H., Olson, J.L., Rennke, H.G., Venkatachalam, M.A., and Brenner, B.M. 1981. Hyperfiltration in remnant nephrons: a potentially adverse response to renal ablation. Am. J. Physiol. 241:F85-F93.

25. Brenner, B.M., Meyer, T.W., and Hostetter, T.H. 1982. Dietary protein intake and the progressive nature of kidney disease: the role of hemodynamically mediated glomerular injury in the pathogenesis of progressive glomerular sclerosis in aging, renal ablation, and intrinsic renal disease. N. Engl. J. Med. 307:652-659.

26. Brenner, B.M. 1985. Nephron adaptation to renal injury or ablation. Am. J. Physiol. 249:F324-F337.

27. Brenner, B.M., and Chertow, G.M. 1994. Congenital oligonephropathy and the etiology of adult hypertension and progressive renal injury. $A m$. J. Kidney Dis. 23:171-175

28. Taal, M.W., and Brenner, B.M. 2000. Renoprotective benefits of RAS inhibition: from ACEI to angiotensin II antagonists. Kidney Int. 57:1803-1817.

29. Anderson, S., Rennke, H.G., and Brenner, B.M. 1986. Therapeutic advantage of converting enzyme inhibitors in arresting progressive renal disease associated with systemic hypertension in the rat. J. Clin. Invest. 77:1993-2000.

30. Ruiz-Ortega, M., Lorenzo, O., Suzuki, Y., Ruperez, M., and Egido, J. 2001. Pro-inflammatory actions of angiotensins. Curr. Opin. Nephrol. Hypertens. 10:321-329.

31. Greene, E.L., Kren, S., and Hostetter, T.H. 1996. Role of aldosterone in the remnant kidney model in the rat. J. Clin. Invest. 98:1063-1068.

32. Abbate, M., et al. 1998. In progressive nephropathies, overload of tubular cells with filtered proteins translates glomerular permeability dysfunction into cellular signals of interstitial inflammation. J. Am. Soc. Nephrol. 9:1213-1224.

33. Eddy, A.A., and Giachelli, C.M. 1995. Renal expression of genes that promote interstitial inflammation and fibrosis in rats with protein-overload proteinuria. Kidney Int. 47:1546-1557.

34. Addis, T. 1948. Glomerular nephritis: diagnosis and treatment. Macmillan. New York, New York, USA. 212 pp.
35. Pedrini, M.T., Levey, A.S., Lau, J., Chalmers, T.C., and Wang, P.H. 1996 The effect of dietary protein restriction on the progression of diabetic and nondiabetic renal diseases: a meta-analysis. Ann. Intern. Med. 124:627-632.

36. Levey, A.S., et al. 1996. Effects of dietary protein restriction on the progression of moderate renal disease in the Modification of Diet in Renal Disease Study. J. Am. Soc. Nephrol. 7:2616-2626.

37. Levey, A.S., et al. 1996. Effects of dietary protein restriction on the progression of advanced renal disease in the Modification of Diet in Renal Disease Study. Am. J. Kidney Dis. 27:652-663.

38. Zatz, R., et al. 1986. Prevention of diabetic glomerulopathy by pharmacological amelioration of glomerular capillary hypertension. J. Clin. Invest. 77:1925-1930.

39. Mogensen, C.E. 1996. Management of the diabetic patient with elevated blood pressure or renal disease. Early screening and treatment programs: albuminuria and blood pressure. In Hypertension: pathophysiology, diagnosis, and management. 2nd edition. J.H. Laragh and B.M. Brenner, editors. Raven Press. New York, New York, USA. 2335-2365.

40. Lewis, E.J., Hunsicker, L.G., Bain, R.P., and Rohde, R.D. 1993. The effect of angiotensin-converting-enzyme inhibition on diabetic nephropathy. The Collaborative Study Group. N. Engl. J. Med. 329:1456-1462.

41. Ravid, M., Lang, R., Rachmani, R., and Lishner, M. 1996. Long-term renoprotective effect of angiotensin-converting enzyme inhibition in non-insulin-dependent diabetes mellitus: a 7-year follow-up study. Arch. Intern. Med. 156:286-289.

42. Kasiske, B.L., Kalil, R.S., Ma, J.Z., Liao, M., and Keane, W.F. 1993. Effect of antihypertensive therapy on the kidney in patients with diabetes: a meta-regression analysis. Ann. Intern. Med. 118:129-138.

43. Lewis, E.J., et al. 2001. Renoprotective effects of the angiotensin-receptor antagonist irbesartan in patients with nephropathy due to type 2 diabetes. N. Engl. J. Med. 345:851-860.

44. Brenner, B.M., et al. 2001. Effects of losartan on renal and cardiovascular outcomes in patients with type 2 diabetes and nephropathy. N. Engl. J. Med. 345:861-869.

45. Maschio, G., et al. 1996. Effect of the angiotensin-converting-enzyme inhibitor benazepril on the progression of chronic renal insufficiency. The Angiotensin-Converting-Enzyme Inhibition in Progressive Renal Insufficiency Study Group. N. Engl. J. Med. 334:939-945.

46. 1997. Randomised placebo-controlled trial of effect of ramipril on decline in glomerular filtration rate and risk of terminal renal failure in proteinuric, non-diabetic nephropathy. The GISEN Group (Gruppo Italiano di Studi Epidemiologici in Nefrologia). Lancet. 349:1857-1863.

47. Ruggenenti, P., et al. 1998. Renal function and requirement for dialysis in chronic nephropathy patients on long-term ramipril: REIN follow-up trial. Gruppo Italiano di Studi Epidemiologici in Nefrologia (GISEN). Ramipril Efficacy in Nephropathy. Lancet. 352:1252-1256.

48. Ruggenenti, P., et al. 1999. In chronic nephropathies prolonged ACE inhibition can induce remission: dynamics of time-dependent changes in GFR. Investigators of the GISEN Group. Gruppo Italiano di Studi Epidemiologici in Nefrologia. J. Am. Soc. Nephrol. 10:997-1006.

49. Ruggenenti, P., et al. 1999. Renoprotective properties of ACE-inhibition in non-diabetic nephropathies with non-nephrotic proteinuria. Lancet. 354:359-364

50. Jafar, T.H., et al. 2001. Angiotensin-converting enzyme inhibitors and progression of nondiabetic renal disease. A meta-analysis of patient-level data. Ann. Intern. Med. 135:73-87.

51. Wright, J.T. 2002. Effect of blood pressure lowering and antihypertensive drug class on progression of hypertensive kidney disease: results from the AASK trial. JAMA. 288:2421-2431.

52. Yusuf, S., et al. 2000. Effects of an angiotensin-converting-enzyme inhibitor, ramipril, on cardiovascular events in high-risk patients. The Heart Outcomes Prevention Evaluation Study Investigators. N. Engl. J. Med. 342:145-153.

53. Taal, M.W., and Brenner, B.M. 2002. Combination ACEI and ARB therapy: additional benefit in renoprotection? Curr. Opin. Nephrol. Hypertens. 11:377-382. 